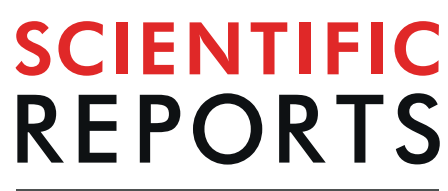

natureresearch

\title{
Capsid-specific removal of circulating antibodies to adeno- associated virus vectors
}

\author{
Berangere Bertin ${ }^{1,6}$, Philippe Veron ${ }^{1,6}$, Christian Leborgne ${ }^{1,6}$, Jack-Yves Deschamps ${ }^{2}$, \\ Sophie Moullec ${ }^{2}$, Yves Fromes $^{3}$, Fanny Collaud ${ }^{1}$, Sylvie Boutin ${ }^{1}$, Virginie Latournerie ${ }^{1}$, \\ Laetitia van Wittenberghe ${ }^{1}$, Benoit Delache ${ }^{4}$, Roger Le Grand ${ }^{4}$, Nathalie Dereuddre-Bosquet ${ }^{4}$, \\ Olivier Benveniste ${ }^{3,5}$, Philippe Moullier ${ }^{2}$, Carole Masurier ${ }^{1}$, Otto Merten ${ }^{1,6}$ \& Federico Mingozzi ${ }^{1,6 *}$
}

Neutralizing antibodies directed against adeno-associated virus (AAV) are commonly found in humans. In seropositive subjects, vector administration is not feasible as antibodies neutralize AAV vectors even at low titers. Consequently, a relatively large proportion of humans is excluded from enrollment in clinical trials and, similarly, vector redosing is not feasible because of development of high-titer antibodies following AAV vector administration. Plasmapheresis has been proposed as strategy to remove anti-AAV antibodies from the bloodstream. Although safe and relatively effective, the technology has some limitations mainly related to the nonspecific removal of all circulating IgG. Here we developed an AAV-specific plasmapheresis column which was shown to efficiently and selectively deplete anti-AAV antibodies without depleting the total immunoglobulin pool from plasma. We showed the nearly complete removal of anti-AAV antibodies from high titer purified human IgG pools and plasma samples, decreasing titers to levels that allow AAV vector administration in mice. These results provide proof-of-concept of a method for the AAV-specific depletion of neutralizing antibodies in the setting of in vivo gene transfer.

Adeno-associated virus (AAV) vectors are currently considered to be one of the most promising platforms for in vivo gene transfer ${ }^{1}$. The lack of pathogenicity of the wild-type virus, the ability to establish long-term transgene expression in post-mitotic cells, and the broad tissue and host range ${ }^{2}$ contributed to the wide adoption of AAV vectors in gene therapy ${ }^{1}$. Recombinant AAV of different serotypes have been successfully tested in human trials ${ }^{3-9}$, and a handful of AAV-based gene therapy products have been approved by the Food and Drug Administration and European Medicine Agency ${ }^{10-12}$. Despite these successes, some important limitations of the AAV technology remain ${ }^{13}$. In particular, pre-existing humoral immunity to the AAV capsid, which develops early in life upon exposure to the wild-type vector ${ }^{14-16}$, represents one of the most important obstacles to successful systemic vector administration in humans ${ }^{17,18}$. Results in mice $^{19}$, non-human primates ${ }^{20}$ and humans ${ }^{5}$ indicate that even low levels of neutralizing antibodies can completely abrogate transduction even when relatively high doses of vector (exceeding $10^{12}$ vector genomes $(\mathrm{vg}) / \mathrm{kg}$ ) are infused through the bloodstream. Moreover, development of high-titer anti-AAV neutralizing antibodies following vector infusion would prevent re-dosing of AAV vectors, in case efficacy is lost over time $\mathrm{e}^{13,21}$.

Pre-existing immunity to AAV resulting from exposure to the wild-type virus is highly prevalent in humans ${ }^{22}$, exceeding two thirds of the population for some AAV serotypes. So far, the approach to the problem of preexisting humoral immunity to AAV in clinical trials has been the exclusion of seropositive subjects from enrollment; however, this solution is far from being optimal, as it results in the exclusion of a significant proportion of otherwise potentially eligible patients. Several strategies have been proposed to address the issue of anti-AAV antibodies ${ }^{17,18}$. Among these, plasmapheresis is an attractive strategy as it represents a safe and clinically established technology broadly available ${ }^{23}$. Plasmapheresis is a process used to selectively remove blood components used to treat

${ }^{1}$ Genethon and INSERM U951, 1 rue de I'internationale, 91000, Evry, France. ${ }^{2}$ Atlantic Gene Therapies, Centre de Boisbonne, ONIRIS, La Chantrerie, BP 40706, 44307, Nantes, France. ${ }^{3}$ Institute of Myology, Sorbonne University and INSERM U974, 105 boulevard de I'Hopital, 75013, Paris, France. ${ }^{4}$ CEA, Université Paris-Sud 11, INSERM U1184, Immunology of Viral Infections and Autoimmune Diseases, IDMIT Department, IBFJ, Fontenay-aux-Roses, 92265, France. ${ }^{5}$ Assistance Publique-Hôpitaux de Paris, 75013, Paris, France. ${ }^{6}$ These authors contributed equally: Berangere Bertin, Philippe Veron, Christian Leborgne,Otto Merten and Federico Mingozzi. *email: fmingozzi@genethon.fr 
a variety of conditions including those caused by the acute overproduction of antibodies (e.g. autoimmunity, transplant rejection), in which removal of pathogenic immunoglobulins results in clinical benefit ${ }^{23}$.

In gene therapy with AAV vectors, plasmapheresis offers several potential advantages, including the fact that it is a relatively simple and risk-free procedure, and the fact that antibody depletion is only transient thus unlikely to result in prolonged immunosuppression. Few studies have explored the effect of plasmapheresis on anti-AAV titers. In humans undergoing plasmapheresis for the management of autoimmunity, repeated sessions of immune absorption reduced circulating anti-AAV antibody levels ${ }^{24}$. The reduction resulted in undetectable neutralizing antibody titers in subjects with low to moderate (1:20) pre-treatment titers, while an only partial decrease in anti-AAV titers was observed in subjects with high-level (e.g. 1:1000) pre-treatment antibodies. In the preclinical setting, non-specific plasma absorption of circulating IgG has also been explored as a strategy to remove anti-AAV antibodies ${ }^{24-27}$.

Despite its advantages, plasmapheresis is also characterized by potential drawbacks associated with the need for repeated cycles over time to completely eliminate detrimental antibodies and the lack of specificity of antibody removal, which inevitably can result in hypogammaglobulinemia, potentially exposing patients to an enhanced risk of infection ${ }^{28}$. In the setting of autoimmunity and transplant, purified human IgG (intravenous immunoglobulin, IVIg) is commonly supplied in combination with plasmapheresis to replenish the pool of circulating antibodies $^{28}$. However, this would not be feasible in the setting of AAV gene transfer due to the fact that IVIg contains high-titer anti-AAV antibodies ${ }^{29}$.

Here, we first tested plasmapheresis as a strategy to lower anti-AAV antibodies in a non-human primate model of AAV gene transfer. We then developed an AAV-specific immune absorption column able to highly-efficiently deplete anti-AAV antibodies from a variety of plasma samples. Depletion of antibodies directed against the capsid did not affect total IgG levels and resulted in a dramatic decrease in neutralizing activity of plasma, even when the starting titer exceeded 1:1000. Matrix optimization and the use of empty AAV particles to capture antibodies further improved the efficacy of the approach as confirmed in vivo in a passive immunization model of gene transfer.

\section{Results}

Plasmapheresis allows for AAV vector readministration in non-human primates. As part of the evaluation of plasmapheresis as a technology to remove circulating anti-AAV neutralizing antibodies (NAbs), a pilot study was conducted in which two cynomolgus macaques received an AAV8 vector encoding for a U7 RNA specific for human dystrophin (AAV8-U7) at a dose of $2.5 \times 10^{11} \mathrm{vg} / \mathrm{kg}$ administered intravenously (Fig. 1a). About 6 weeks later, when AAV NAb titers stabilized, one animal (labeled as Plasmapheresis) underwent 3 cycles of plasmapheresis 48 hours apart, which resulted in a 100-fold decrease in anti-AAV NAb titers, down to levels compatible with efficient AAV vector transduction ${ }^{20}$. Following the procedure, $2.5 \times 10^{13} \mathrm{vg} / \mathrm{kg}$ of an AAV8 vector encoding for human secreted embryonic alkaline phosphatase (AAV8-huSEAP) were given intravenously. After each plasmapheresis cycle, the animal received an infusion of plasma from compatible animals with negative AAV NAbs titer to avoid excessive depletion of circulating immunoglobulins. The AAV8-huSEAP vector at the same dose was also administered to the immunized control animal which did not receive plasmapheresis (labeled as Control) and to a naïve animal with undetectable anti-AAV8 NAb titer (labeled as Naïve). All animals were followed for 70 days post AAV8-huSEAP administration and then sacrificed. Vector genome copy number (VGCN) specific for the AAV8-huSEAP vector was then evaluated in a variety of tissues (Fig. 1b). As previously published $^{25,27}$, the Plasmapheresis animal displayed the highest VGCN in all tissues, closely followed by the Naïve animal. Conversely, as expected, the Control animal had the lowest VGCN in most tissues except for spleen (Fig. 1b), consistent with the expected biodistribution of AAV vectors in animals presenting anti-AAV antibodies at the time of vector administration ${ }^{29,30}$. The liver appeared to be the highest targeted organ, with VGCN in the Plasmapheresis animal $\sim 1 \log$ higher than the Control animal (Fig. 1b). Circulating levels of huSEAP in blood also confirmed the VGCN findings (Supplementary Fig. 1).

Anti-AAV antibody levels were also consistent with the VGCN results (Fig. 1c-e). Anti AAV-IgG and NAb titers decreased in the Plasmapheresis animal at each cycle of plasma absorption, down to levels close to preimmunization (Fig. 1c,d). Titers then increased again following the administration of the AAV8-huSEAP vector similar levels in all animals (Fig. 1c,d). As expected, IgM titers had an overlapping profile in all animals, characterized by a transient increase after each AAV vector administration (Fig. 1e).

These results confirm that plasmapheresis is a potentially useful technology to reduce pre-existing anti-AAV NAbs allowing for vector readministration, although it requires IgG supplementation to lower the risk of infection derived from the depletion of circulating antibodies.

Grafting of capsid onto an NHS-Sepharose column results in efficient anti-AAV antibody capture. Based on the promising results in vivo in non-human primates, we wanted to develop an improved technology to capture specifically anti-AAV antibodies. Toward this aim, we developed a series of chromatography columns based on commercially available matrices that we used to graft AAV capsid (Fig. 2a). Initially, we evaluated non-compressible chromatography matrices because they allow a relatively easy translation to larger scale, these included EPO-POROS (Epoxy activated chromatography support) in which the AAV capsid was grafted via EPO chemistry or crosslinking with formaldehyde. Both methods resulted in efficient capture of anti-AAV antibodies, although formaldehyde treatment resulted in lower retention of IgG (Supplementary Fig. 2). Based on published literature on plasmapheresis ${ }^{31-33}$, we then focused our attention on Sepharose, a compressible chromatography support. In a preliminary experiment, $3.4 \times 10^{12} \mathrm{vg}$ of AAV8 particles were crosslinked to $5 \mathrm{ml}$ $\mathrm{N}$-Hydroxysuccinimidyl (NHS)-Sepharose. Leakage of vector genomes from the column was monitored using quantitative real-time PCR, showing only minimal leakage $\left(\sim 1 \times 10^{8} \mathrm{vg}\right)$ of viral particles after loading and four washes with PBS and sodium citrate followed by three empty runs (Supplementary Fig. 3). Next, three loads of intravenous immunoglobulin (IVIg, pooled human IgG containing anti-AAV IgG) or plasma were applied to 


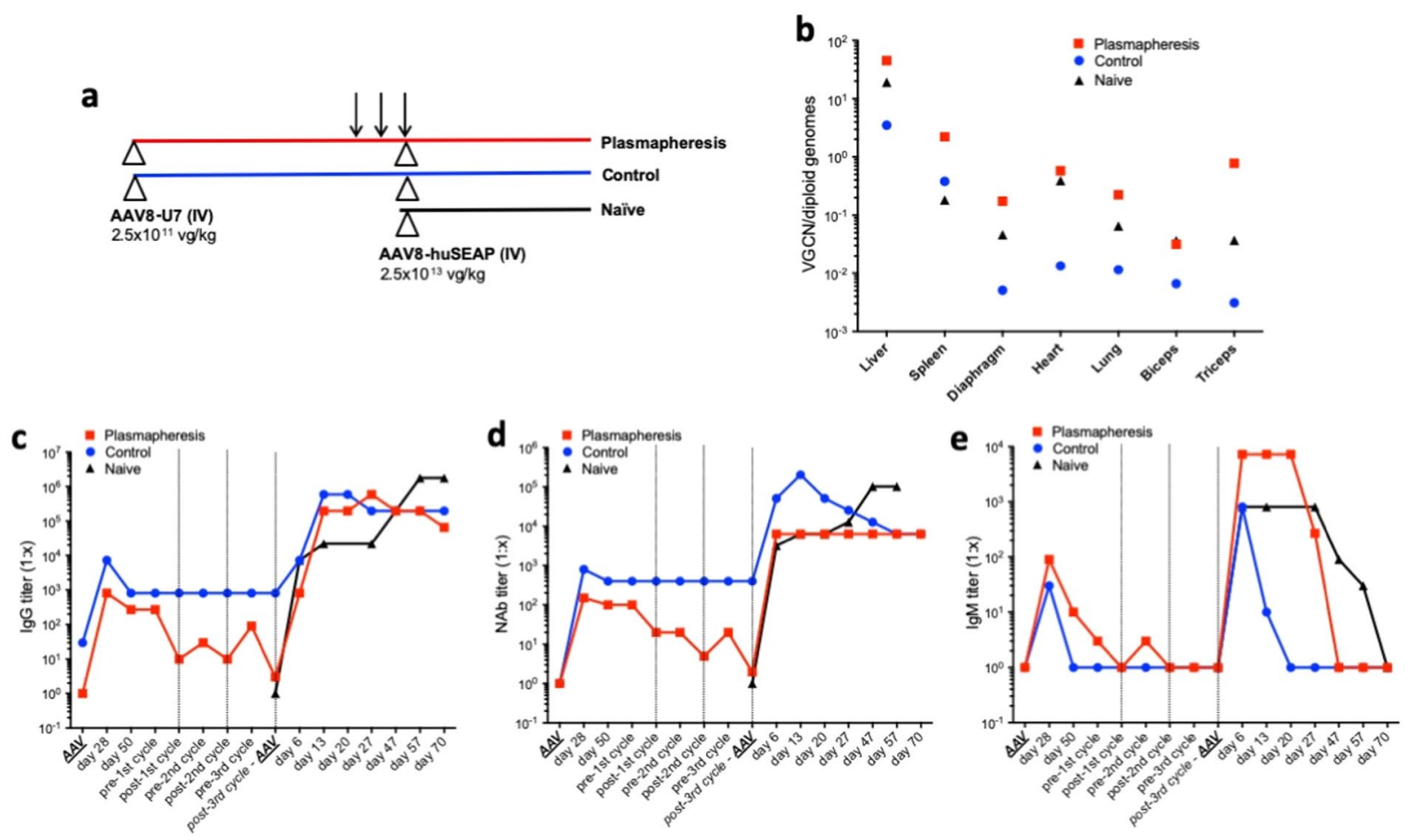

Figure 1. Plasmapheresis allows for AAV vector readministration in non-human primates. (a) Experimental design. Two animals received an AAV8-U7 vector intravenously at a dose of $2.5 \times 10^{11} \mathrm{vg} / \mathrm{kg}$ (Plasmapheresis and Control), 6 weeks later one animal (Plasmapheresis) was subjected to 3 cycles of plasmapheresis at a 2-day interval (arrows). Both animals and a third naïve control animal (Naïve) were then infused with an AAV8huSEAP vector at a dose of $2.5 \times 10^{13} \mathrm{vg} / \mathrm{kg}$. Triangles indicate AAV vector administrations. (b) Vector genome copy number (VGCN) per diploid genome in various tissues collected at sacrifice. (c-e) Anti-AAV8 IgG (c), $\mathrm{NAb}(\mathbf{d})$, and $\operatorname{IgM}(\mathbf{e})$ levels over time. AAV in the $\mathrm{x}$ axis indicate the timing of AAV vector administration. Vertical dotted lines represent the timing of plasmapheresis cycles. Titers are reported as 1:x dilution.

the column at a flow rate of $0.5 \mathrm{ml} /$ minute $(0.1 \mathrm{CV} /$ minute), and retention of capsid IgG was estimated using an anti-AAV8 IgG ELISA assay before loading, on flow through and after elution of the column (Fig. 2b). Retention $>70 \%$ for IVIg and $>80 \%$ for plasma of anti-AAV8 IgG was measured with different loads of IVIg as well as different volumes and dilutions of plasma (Fig. 2b), demonstrating efficient binding of anti-AAV8 antibodies to the AAV-Sepharose column.

Using the prototype column, we then evaluated the role of the density of the ligand on the efficiency of IgG retention (Fig. 2c). Three AAV8 densities were tested using a $5 \mathrm{ml}$ volume of NHS-Sepharose, $9.3 \times 10^{11}$, $1.1 \times 10^{13}$, and $6.0 \times 10^{13} \mathrm{vg} /$ column. $72 \mathrm{mg}$ of IVIg or $2 \mathrm{ml}$ of human plasma positive for anti-AAV8 antibodies were loaded onto the columns and retention rate measured by ELISA. At the lowest AAV8 concentration, $9.3 \times 10^{11} \mathrm{vg} /$ column, retention of anti-AAV8 IgG was about $70 \%$ for IVIg. Possibly due to the presence of several proteins which may bind to $\mathrm{AAV}^{34,35}$, thus potentially interfering with the binding to the AAV capsid grafted to the column, only $45 \%$ of AAV8 IgG were retained when plasma was loaded to the column. Higher AAV8 concentrations resulted in better retention of antibodies, with $>90 \%$ retention observed for both IVIg and plasma with the column containing $6.0 \times 10^{13} \mathrm{vg} /$ column AAV8 (Fig. $2 \mathrm{c}$ ).

Next, we evaluated the effect of flow rate on the anti-AAV8 retention rate. Using three columns with $1 \mathrm{ml}$ of resin grafted with $9.3 \times 10^{11}, 3.4 \times 10^{12}$, or $6 \times 10^{12} \mathrm{vg}$ of AAV8, anti-AAV8 IgG retention was determined at flow rates of $0.1,0.25$, and $0.5 \mathrm{ml} / \mathrm{min}(0.02,0.05$, and $0.1 \mathrm{CV} / \mathrm{min}$, Fig. $2 \mathrm{~d})$. As expected, higher flow rates resulted in lower retention of IgG in a setting a single passage of the IgG-containing fluid through the column. Repeated passages of IVIg through the same column or sequential passages through two columns enhanced removal of AAV8-specific IgG at any given flow rate (not shown).

These results support the feasibility of removing anti-AAV antibodies from plasma, they also establish some of the critical parameters affecting IgG retention efficiency.

Empty AAV capsids retain IgG more efficiently than full capsids. In view of a possible use of an AAV-specific plasmapheresis column in humans, therefore to avoid the use of pharmacologically active AAV vectors in the plasmapheresis column, we tested whether empty capsids could be used to capture anti-AAV IgG. Multiple IgG retention experiments were performed with columns containing $2 \times 10^{12}$ particles of either full ( $n=8$ runs) or empty ( $n=5$ runs) AAV capsids (Fig. 3a), showing a significantly more efficient retention of anti-AAV8 IgG with empty vs. full capsids $(p<0.01 \mathrm{t}$ test) at a flow rate of $0.25 \mathrm{ml} / \mathrm{min}$. To better investigate these findings, serial dilutions of both IVIg and human plasma were used in capture assays in which plates were coated 


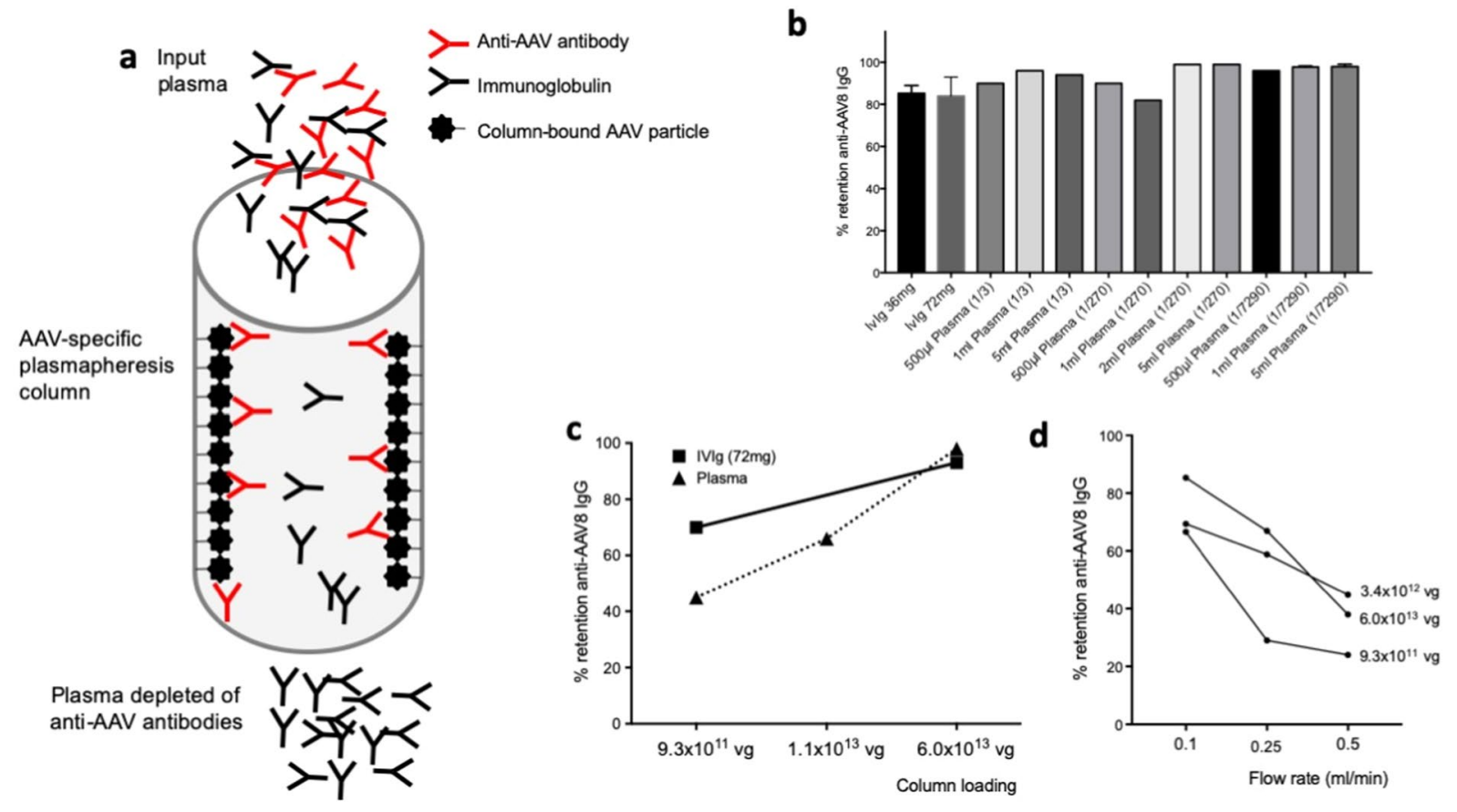

Figure 2. AAV-specific column for the removal of anti-capsid IgG from plasma. (a) Diagram of the column. AAV capsid particles are grafted to the column matrix and allow for binding and removal of IgG specific to the capsid without affecting total antibody levels. (b) Anti-AAV8 IgG retention rate of IVIg and plasma at various concentrations and volumes. A flow rate of $0.1 \mathrm{ml} /$ minute was used in all experiments. For the IVIg retention experiment, $\mathrm{n}=3$ runs. For $1 \mathrm{ml}$ and $5 \mathrm{ml}$ plasma at $1 / 7290$ dilution, $\mathrm{n}=5$ runs; shown is average, error bars represent the standard error of the mean. (c) Effect of AAV capsid loading on anti-AAV8 IgG retention. Various amounts of AAV8 capsid were used to bind to the NHS-Sepharose matrix and retention of AAV8 IgG measured after IVIg or seropositive plasma loading. (d) Effect of flow rate on anti-AAV8 IgG retention. AAV8 IgG retention tested with 3 columns containing various amounts of AAV8 capsid at 3 different flow rates.

with either empty or full AAV8 capsid (Fig. 3b,c). Detection of bound IgG with a secondary antibody confirmed the higher affinity of anti-AAV8 IgG for empty capsids. A series of similar tests with different preparations of empty and full capsids $(\mathrm{n}=6 \mathrm{each})$ was then performed with IVIg using plates coated with $2 \times 10^{10}$ or $2 \times 10^{11}$ capsid particles/ml (Fig. 3d,e). In limiting conditions, i.e. in the presence of lower amounts of capsid coated onto the plates $\left(2 \times 10^{10}\right.$ capsid particles/ml), empty capsids showed a higher, although not statistically significant, binding affinity for anti-AAV antibodies (Fig. 3d), which was not seen at higher capsid concentrations (Fig. 3e).

These results suggest that empty capsids bind anti-capsid antibodies more efficiently than full, potentially representing a better biological material for the development of AAV-specific plasmapheresis columns.

AAV plasmapheresis columns specifically remove anti-AAV antibodies from plasma. After optimization of the plasmapheresis column conditions, plasma samples of different origin were used to test efficiency and specificity of removal of capsid antibodies. Plasma was specifically used in these experiments as it was considered more representative of the clinical setting compared to that of purified IgG present in IVIg. First, plasma from a dog previously immunized with an AAV8 vector and displaying a baseline NAb titer of $>1: 10,000$ was tested (Fig. 4a,b). The column was initially loaded with plasma and the flow through (FT) fractions of plasma were collected for analysis, showing lack of anti-AAV8 IgG and presence of total IgG. Conversely, when the column was eluted (E), only anti-AAV8 antibodies were detectable in the fractions collected (Fig. 4a). Accordingly, and in agreement with the published correlation between IgG and NAb titers ${ }^{36}$, only the elution fractions showed the presence of neutralizing antibodies against AAV8 (Fig. 4b). Consistent with a calculated $\sim 30$-fold dilution factor of the input seropositive plasma, the anti-AAV8 NAb titer in the elution fractions of the column peaked at 1:316 (Fig. 4b). Next, plasma from a non-human primate previously dosed with an AAV8 vector (Fig. 4c) and from a healthy donor pre-exposed to wild type AAV (Fig. 4d) were tested, showing similar specific retention of anti-capsid antibodies. NAb titers in the human plasma sample, which had a baseline NAb titer of 1:1,000, dropped to 1:3.16 in the FT fractions (after retention of the anti-AAV8 Ig), while NAb titers of 1:10 were found in the elution fractions. To compare these results with a column that was not antigen-specific, a protein A column was tested with a seropositive plasma sample (Fig. 5). As expected, virtually no IgG was released in the flow through (FT) at the time of passage of plasma through the column, while both total IgG and anti-AAV8 IgG were found in the fractions collected at the time of elution of the column (Fig. 5).

These results support the efficacy and specificity of AAV8 plasmapheresis columns in the removal of anti-capsid antibodies from plasma, even in the presence of high-titer antibodies derived from active immunization with AAV vectors. 


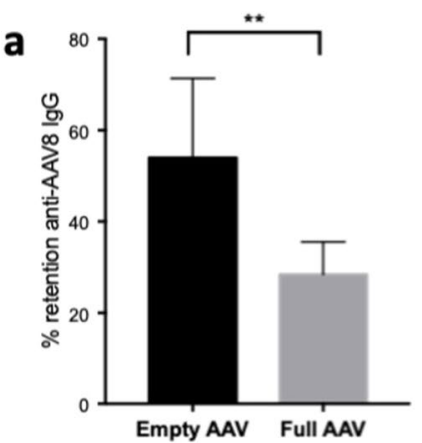

b

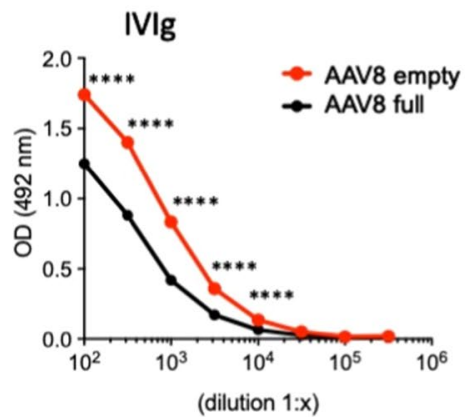

C

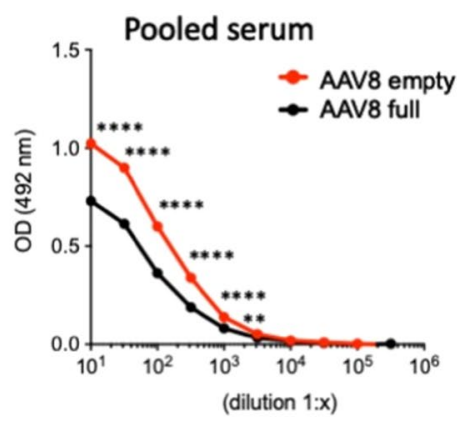

d

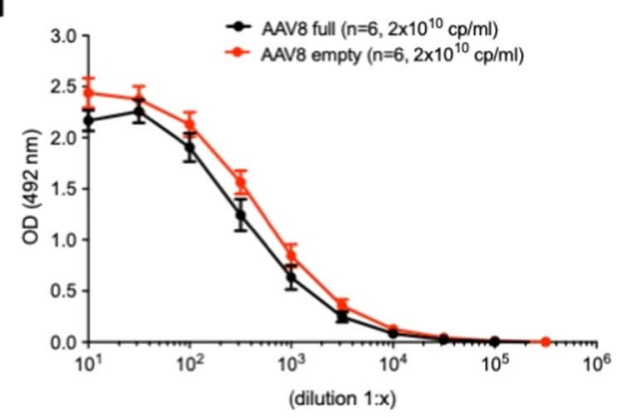

e

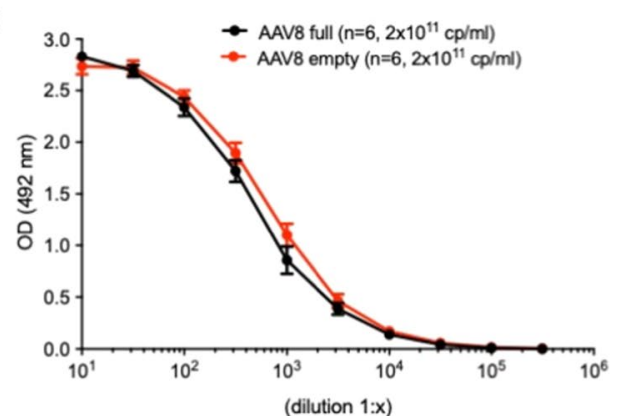

Figure 3. Binding of anti-AAV8 IgG to empty and full capsids. (a) AAV8 IgG retention following loading of $36 \mathrm{mg}$ of IVIg loading onto NHS-Sepharose columns loaded with the same amount $\left(2 \times 10^{12}\right.$ capsid particles, purified from the same AAV preparation) of empty vs. full capsids (empty AAV, $n=5$ runs; full AAV, $n=8$ runs). ${ }^{*} p<0.01$ two-tailed unpaired t test. (b,c). ELISA assay to detect anti-AAV8 IgG in IVIg (b) or pooled human serum $(\mathbf{c})$ at various dilutions. Plates were coated with $2 \times 10^{10}$ capsid particles $(\mathrm{cp}) / \mathrm{ml}$ of empty or full AAV8 capsid. (d,e). ELISA assay to detect anti-AAV8 IgG in IVIg using plates coated with either $2 \times 10^{10}$ (d) or $2 \times 10^{11}(\mathbf{e}) \mathrm{cp} / \mathrm{ml}$ of empty or full AAV8 capsids deriving from $\mathrm{n}=6$ different preparations each. Error bars represent the standard error of the mean. Statistical analyses performed by RM two-way ANOVA, Sidak's multiple comparisons test in panels $(\mathrm{b}, \mathrm{c})$ and ordinary two-way ANOVA, Sidak's multiple comparisons test in panels $(\mathbf{d}, \mathbf{e}) . * * * * p<0.0001 ; * * p<0.01$

Column-mediated removal of anti-AAV8 IgG allows for efficient liver transduction in vivo. To confirm our findings in vivo, we used a previously published passive immunization mouse model ${ }^{37}$. Seropositive plasma was passed through an AAV-Sepharose column and the different fractions of the column were subsequently infused intravenously in C57BL/6 mice. The day after C57BL/6 mice $(n=5 / 6$ per group) received an AAV8 vector encoding for coagulation factor IX (AAV8-hF.IX) at a dose of $5 \times 10^{10} \mathrm{vg} / \mathrm{mouse}$. Animals were followed up for 4 weeks (Fig. 6a). Before column treatment, the plasma sample used for the passive immunization experiment presented a high NAb titer (Plasma, NAb titer 1:1000), which decreased to undetectable in the flow through (FT, NAb titer < 1:1) of the column (Fig. 6b). As observed in previous experiments, the anti-AAV8 NAbs were then found in the elution fraction of the column at a titer decreased due to the $\sim 30$-fold dilution of the sample (E, NAb titer 1:31.6, Fig. 6b).

Animals were passively immunized with Plasma, FT or E fractions, or PBS as control and after vector delivery hF.IX transgene levels in plasma were determined by ELISA. No transgene expression was observed in animals receiving untouched plasma, while animals receiving the FT fraction of the column had significantly higher transgene expression levels (Fig. 6c). Accordingly, vector genome copy number (VGCN) in livers collected at day 28 post vector infusion showed significantly higher levels of transduction of hepatocytes in FT-treated mice compared to Plasma or E treated animals (Fig. 6d).

These results confirm, in an in vivo model of passive immunization, that pretreatment of plasma with an AAV-specific antibody binding column results in rescue of liver transduction following AAV vector administration.

\section{Discussion}

Pre-existing anti-AAV antibodies are one of the main obstacles to the efficient systemic delivery of AAV vectors. Antibodies originated from exposure to wild-type AAV, or deriving from administration of AAV vectors, can in fact neutralize AAV vectors, resulting in lack of efficacy ${ }^{5}$. Consequent to humoral immune responses to AAV, a significant proportion of seropositive participants is excluded from AAV vector-based trials, while vector redosing, in case needed, is virtually impossible without immunomodulation at the time of gene transfer ${ }^{38,39}$. Here, we initially tested the efficiency of plasmapheresis in the removal of anti-AAV antibodies in non-human primates. Like previously described ${ }^{25}$, we showed rescue of tissue transduction following systemic AAV vector, although IgG-containing plasma supplementation was necessary to maintain the levels of total circulating IgG sufficient to 
a

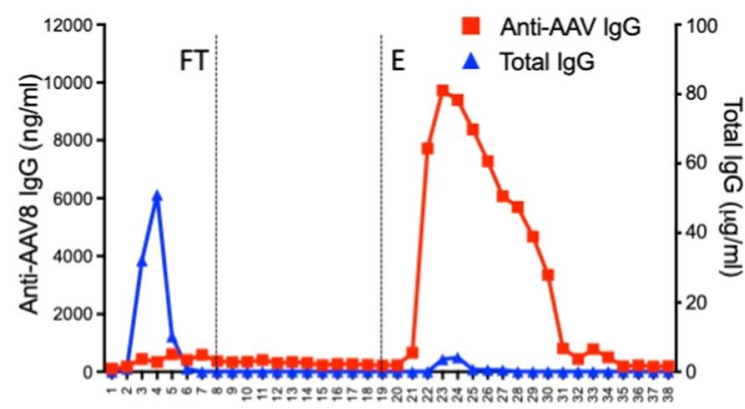

Column fraction

C

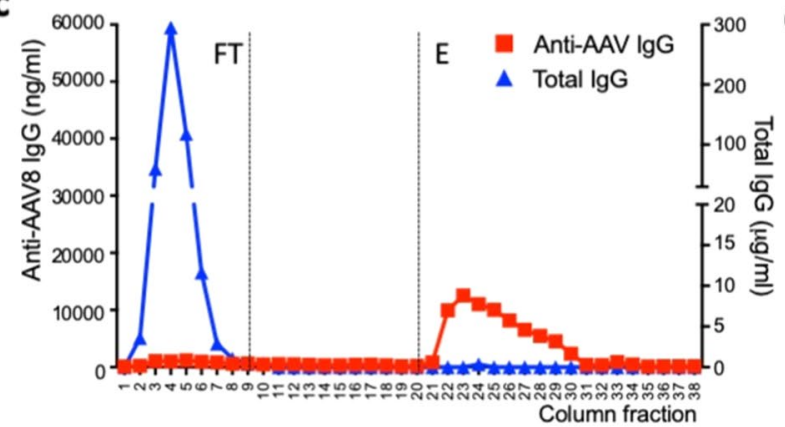

b
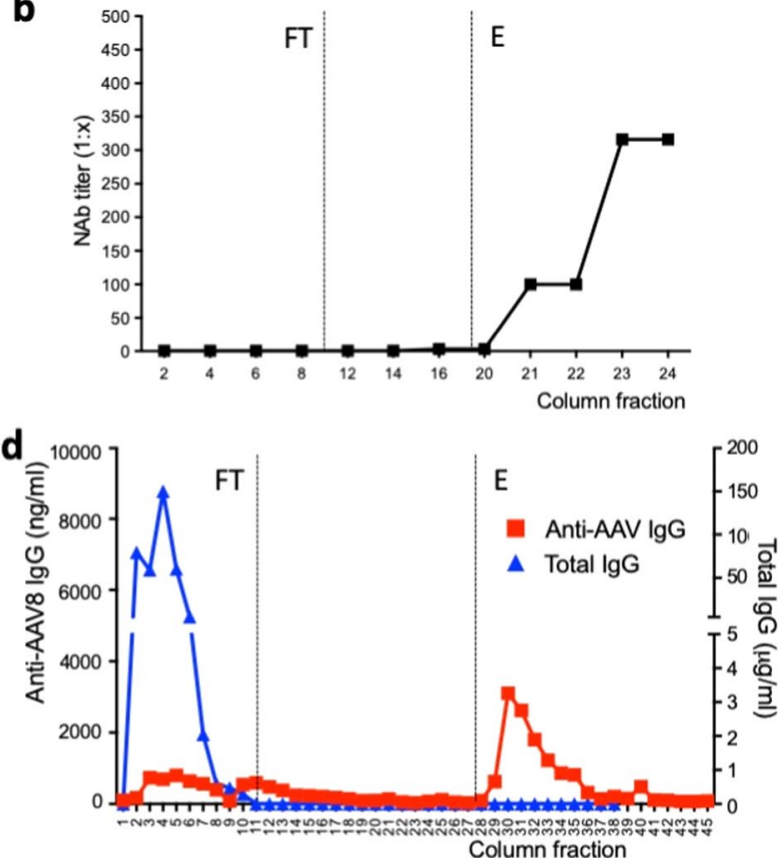

Figure 4. Antibody retention profile of AAV8-Sepharose columns. All columns contained full AAV capsids $\left(2 \times 10^{12} \mathrm{vg} / 1 \mathrm{ml}\right.$ of resin) (a) Anti-AAV8 IgG (red) and total IgG (blue) levels measured by ELISA in flow through (FT) and elution (E) fractions of an AAV8-Sepharose column after loading seropositive dog plasma $(5 \mathrm{ml})$. (b) Anti-AAV8 NAb profile of the same dog sample across selected fractions from FT and E. NAb titers are reported as reciprocal dilution (1:x). Starting NAb titer before column treatment was $>1: 10,000$. (c,d) AntiAAV8 IgG (red) and total IgG (blue) levels measured in non-human primate (c) and human (d) plasma (loading volume $5 \mathrm{ml})$.

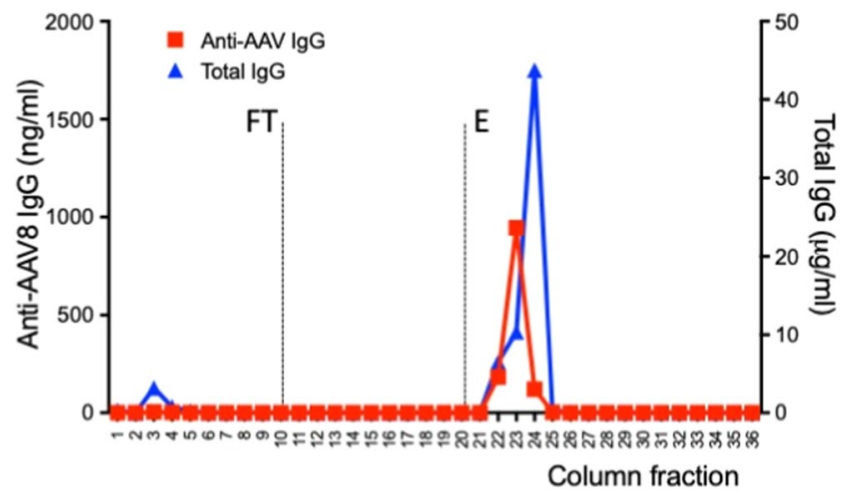

Figure 5. Antibody retention profile of a protein A column. Anti-AAV8 IgG (red) and total IgG (blue) levels measured by ELISA in flow through (FT) and elution (E) fractions of a protein A column after loading seropositive dog plasma $(5 \mathrm{ml})$.

prevent infections. Next, to improve the safety and the efficacy of plasmapheresis to remove anti-capsid antibodies, we developed an AAV-specific plasmapheresis column for the selective removal of anti-AAV antibodies from the bloodstream. By optimizing column conditions, and defining the parameters critical for antibody absorption, we achieved nearly complete removal of vector-specific antibodies from pooled purified IgG (IVIg) preparations and from various plasma samples after a single depletion passage. We showed that removal of capsid antibodies was selective, as the total pool of circulating IgG was not depleted; furthermore, we showed that plasma samples passed through the column lost their ability to neutralize AAV vectors in vivo in a murine model of passive immunization.

Plasmapheresis is routinely used for the physical removal of antibodies from the circulation. The advantage of plasmapheresis over pharmacological approaches targeting B cells ${ }^{40}$ is the high efficiency in the removal of antibodies and the fact that the effect is only transient as it does not involve targeting antibody producing cells with immunosuppressive drugs. The technology is indicated in several autoimmune diseases and to prevent 


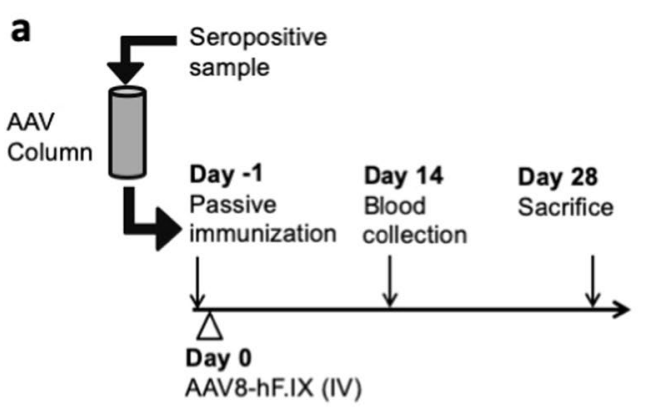

C

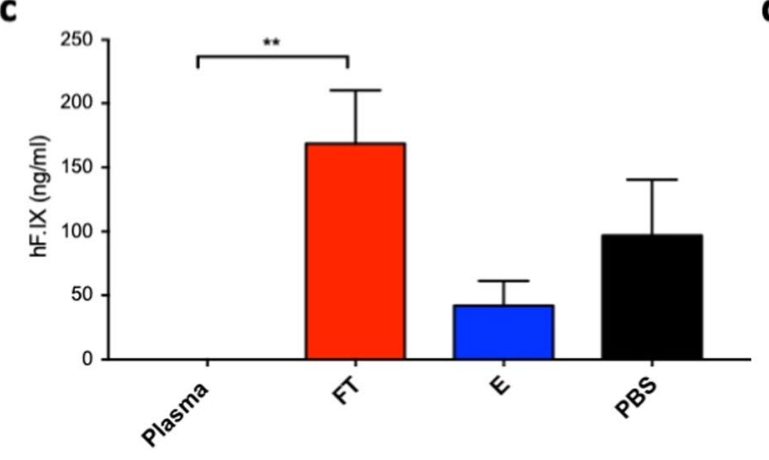

b

\begin{tabular}{|c|c|}
\hline Sample fraction & $\begin{array}{c}\text { Anti-AAV8 } \\
\text { NAb (1:x) }\end{array}$ \\
\hline $\begin{array}{c}\text { Untouched plasma } \\
\text { (Plasma) }\end{array}$ & $1: 1000$ \\
\hline Flow through (FT) & $<1: 1$ \\
\hline Elution (E) & $1: 31.6$ \\
\hline PBS & $<1: 1$ \\
\hline
\end{tabular}

d

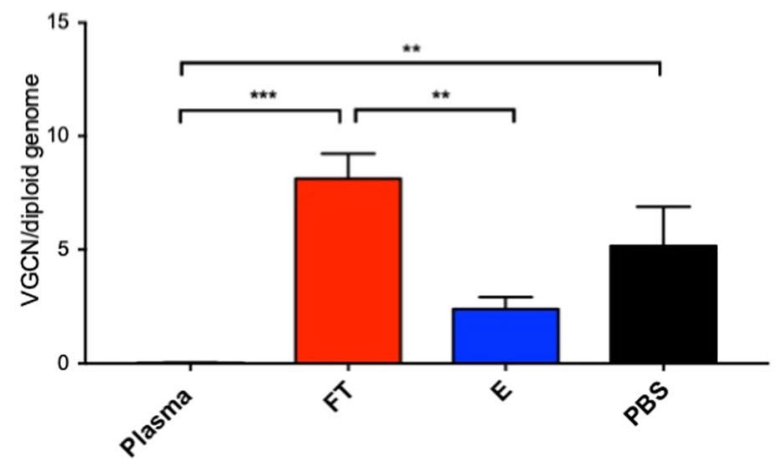

Figure 6. In vivo testing of removal of anti-capsid antibodies with the AAV8-Sepharose column. (a) Experimental design. A seropositive sample from a dog previously dosed with an AAV8 vector and displaying a titer of 1:1000 was subjected to column-based depletion of anti-AAV8 antibodies. Untouched plasma (Plasma), flow through (FT), elution (E) or PBS as control (PBS) were delivered to C57BL/6 mice (100 ul/mouse, $\mathrm{n}=5 / 6$ per treatment group) on day -1 . On day $05 \times 10^{10} \mathrm{vg} /$ mouse of AAV8-hF.IX vector were delivered intravenously. Plasma levels of hF.IX were measured at day 14 and vector genome copy number (VGCN) were measured in liver at day 28. (b) Anti-AAV8 NAb profile of samples delivered to mice on day -1. (c) hF.IX plasma levels in mice at day 14 post vector administration. (d) VGCN per diploid genome in liver of mice at sacrifice. Results in (c,d) are shown as average, error bars represent the standard error of the mean. ${ }^{* *} p<0.01$; $* * * p<0.001$ one-way ANOVA.

antibody-mediated graft rejection ${ }^{23}$. Few publications can be found in the literature on the use of plasmapheresis in the context of AAV gene transfer, including preclinical studies in non-human primate ${ }^{25,26}$ suggesting that the technology is effective in removing anti-AAV antibodies. To this aim, Monteilhet and colleagues ${ }^{24}$ showed that repeated cycles of plasma absorption in autoimmune disease patients results in the reduction of anti-AAV antibody titers, although this requires several subsequent sessions to efficiently eliminate both vascular and extravascular antibodies to AAV. Interestingly, in this study 5 cycles of plasmapheresis were not effective in completely eliminating high-titer neutralizing antibodies ${ }^{24}$, thus suggesting the need for more cycles of plasma absorption. While in theory it is possible to repeat immune absorption cycles until all antibodies are eliminated, this would be associated with prolonged periods of hypogammaglobulinemia and potential exposure to infections. The strategy presented here, based on the selective removal of anti-AAV antibodies, present a clear improvement over the conventional nonspecific technology as it would allow for repeated anti-AAV antibody depletion cycles with no effect on total IgG levels. In the context of conventional plasmapheresis, a decrease of IgG levels below a critical level would require supplementation with pooled human IgG, which by default contain high titer anti-AAV NAbs. One possible solution to the issue, which was also implemented here to supplement IgG in the non-human primate undergoing conventional plasmapheresis, is the supplement of IgG from AAV seronegative subjects, or else the development of AAV antibody-negative IgG pools. However, both approaches are unlikely to be feasible, as anti-AAV antibodies are highly prevalent in humans ${ }^{22}$.

One somewhat unexpected result obtained in the current study is the higher affinity of anti-AAV antibodies for empty capsids. The explanation of the finding not entirely unclear, although it is known that empty and full capsid are not identical, based on electron microscopy images of empty and full capsids ${ }^{41}$ and on the fact that it is possible to design purification methods able to discriminate between empty and full capsids ${ }^{42}$, suggesting both some underlying biological and structural differences driving the different binding to circulating antibodies. One possibility is that, due to the absence of the DNA genome, empty capsids could have a higher flexibility allowing a more intense antigen-antibody interaction than full AAV capsid particles. In this context, Carrasco and colleagues ${ }^{43}$ using the minute virus of mice (MVM, a parvovirus) were able to show specific protein-DNA interactions in the case of full capsids owing to an anisotropic increase in the mechanical capsid stiffness. Whether a similar model apply also to interactions of antibodies with the AAV capsid remains to be elucidated, along with binding affinity studies. Clearly, empty capsids are the most suitable material to be used to develop a plasmapheresis column, as they are inert due to the lack a genome. 
While the data presented here are promising, additional work will be needed to include the technology presented here in a plasmapheresis device and to test the strategy in large animal models that are natural host for $\mathrm{AAV}$, like non-human primates $^{44}$, to mimic the context of AAV immunity found in humans and the actual plasmapheresis conditions used in the clinic. Critical parameters, like the optimal quantity of capsid antigen required to efficiently capture anti-AAV Ig, will also need to be defined. Furthermore, it would be useful to identify protocols in which the current technology is combined with an immunomodulatory regimen to increase the efficiency of the approach and thus achieve clearance of anti-AAV antibodies more rapidly than with plasmapheresis alone ${ }^{45}$. While the current proof-of-concept work demonstrated the feasibility of the approach in the context of one specific serotype, the development of column containing multiple AAV serotypes, therefore with different specificities, could potentially represent a universal device for antibodies directed to different AAVs. Additionally, as data on domains within the AAV capsid that are also epitopes of neutralizing antibodies become available, it may be possible to develop an AAV-free plasmapheresis column only based on specific peptides.

One open question about the technology described here is about the potential risk of triggering immunotoxicities associated with reactivation of memory cytotoxic $\mathrm{T}$ responses directed against transduced cells ${ }^{18}$. In the setting of AAV vector redosing, immunosuppression with corticosteroids ${ }^{3,8}$, other T cell-targeting drugs ${ }^{46}$, or the use of agents targeting both $\mathrm{B}$ and $\mathrm{T}$ cell responses to $\mathrm{AAV}^{39}$, used in combination with anti-AAV Ig depletion, may be required. Given the lack of correlation between humoral and cell-mediated immunity in the context of natural immunity to wild-type $\mathrm{AAV}^{36,47,48}$, the need for immunomodulation in the setting of eradication of naturally occurring anti-AAV antibodies is less understood.

In summary, here we presented a strategy based on AAV-specific plasmapheresis for the selective removal of circulating antibodies that can neutralize AAV vectors. The approach appeared to remove antibodies efficiently and specifically, without affecting total IgG levels. This work provides proof-of-concept of the feasibility of the approach and its potential using as an enabler of systemic gene transfer in vivo when anti-vector antibodies are present.

\section{Materials and Methods}

Specimens and animal experiments. All plasma samples of human origin were purchased from the French blood bank (Etablissement Français du Sang, EFS, Rungis, France). Intravenous immunoglobulin (IVIg) was purchased from LFB Biomedicaments (Courtaboeuf, France), the same lot of IVIg was used across all studies shown in this manuscript. All samples from pre-immunized animals came from previous studies approved by the local ethical committees.

Male C57BL/6 mice (males, 8-10 weeks of age) were obtained from Charles River (St. Germain Nuelles, France). Mouse studies were performed in accordance to the current European legislation on animal care and experimentation (2010/63/EU) and approved by the institutional ethical committee of the Centre d'Exploration et de Recherche Fonctionnelle Expérimentale (protocol number APAFIS 3055-20151019213299180).

Adult cynomolgus macaques (Macaca fascicularis) were imported from Noveprim Europe and housed at the facilities of the Centre de Boisbonne, Ecole Nationale Vétérinaire de Nantes (ONIRIS, Nantes). The plasmapheresis protocol was reviewed by the Ethical Animal Committee and registered by the French Research Ministry. Blood collection for the isolation of seronegative plasma was approved by the Ethical Committee number 44 of CEA (French Alternative Energies and Atomic Energy Commission), study number A13_017.

AAV vectors. The AAV8 vector expressing human secreted embryonic alkaline phosphatase (AAV8-huSEAP) and the AAV8-U7 vector ${ }^{49}$ were produced in a baculovirus system ${ }^{50}$. The AAV8 vector encoding for human factor IX (AAV8-hF.IX) was previously described ${ }^{37}$, this vector and the preparations of full and empty vector particles were made as previously described ${ }^{51}$. All vectors were titrated using a quantitative real-time PCR assay. Empty AAV capsids were quantified using SDS-PAGE after Sypro Ruby staining (Molecular Probes, Thermo Fisher Scientific, Waltham, MA). Gel images were acquired on a gel reader G:box HR (Syngene, Frederick, MD) and the bands corresponding to the VP3 capsid protein quantified against a standard of known concentration using the software Image studio light (LI-COR Biosciences, Lincoln, NE).

Preparation of the column matrices. Two different columns matrices, one based on the compressible NHS-Sepharose (HiTrap NHS activated, N-hydroxysuccinimide activated, GE HealthCare, St. Quentin en Yvelines, France) and one based on the incompressible EPO-POROS 50 resin (POROS EP450, $50 \mathrm{~nm}$, Thermo Fisher Scientific, Waltham, MA), were initially evaluated in this study. For the NHS-Sepharose, the ligand density was $10 \mu \mathrm{mol}$ ligand/ml of gel according the manufacturer directions; for the EPO-POROS support, the ligand density was determined empirically. Full or empty AAV particles formulated in PBS were grafted to the supports following the manufacturers' recommendations. For EPO-POROS, $3.7 \times 10^{11}$ particles of AAV8 capsid were added to $1 \mathrm{ml}$ of resin. Coupling was performed at $4{ }^{\circ} \mathrm{C}$ for 60 hours. Residual active sites were inactivated by incubation with ethanolamine ( $1 \mathrm{M}$ in a $0.5 \mathrm{M}$ phosphate buffer, $\mathrm{pH} 9.0$ ) for 2 hours at room temperature followed by washing with PBS. In order to reduce potential leaching of coupled AAV particles, crosslinking with formaldehyde (37\% diluted $1 / 200$ in PBS) was performed at $4{ }^{\circ} \mathrm{C}$ for 15 days. Then, residual formaldehyde was inactivated by incubation with Na-meta-bisulphite (3.75\%, diluted 1/100 in PBS) for 2 hours at room temperature, followed by washing with PBS. For NHS-Sepharose, different amounts of AAV8 capsid ranging from $9.3 \times 10^{11}$ to $6.0 \times 10^{13}$ particles were coupled with $5 \mathrm{ml}$ of HiTrap NHS support. After 1 hour of incubation at room temperature, residual active sites were inactivated by incubation with ethanolamine for 30 minutes at room temperature followed by washing with PBS. At the time of grafting of AAV particles to a column, four washes with PBS and sodium citrate at $\mathrm{pH} 4$ were performed to eliminate unbound capsid (Supplementary Fig. 3). 
Column conditions for removal of anti-AAV antibodies from biological samples. All chromatography runs were performed on an ÄKTA explorer chromatography system (GE HealthCare, Uppsala, Sweden). UV $(280 \mathrm{~nm}, 260 \mathrm{~nm}), \mathrm{pH}$, conductimetry $(\mathrm{mS})$ and flow rate were monitored during each run. For the EPO-POROS columns, the tests were performed with either a 1.4-fold dilution of IVIg (to a concentration of IgG of $35.7 \mathrm{mg} / \mathrm{ml})$ or undiluted plasma. A flow rate of $0.5 \mathrm{ml} / \mathrm{min}(0.5$ column volume $(\mathrm{CV}) / \mathrm{min})$ was applied. For the NHS-Sepharose columns, various volumes of diluted IVIg $(35.7 \mathrm{mg} / \mathrm{ml})$ or undiluted plasma samples were used as indicated in the results section. Different flow rates ranging from $0.5 \mathrm{ml} / \mathrm{min}(0.1$ $\mathrm{CV} / \mathrm{min})$ to $0.1 \mathrm{ml} / \mathrm{min}(0.02 \mathrm{CV} / \mathrm{min})$ were initially tested and a rate of $0.1 \mathrm{ml} / \mathrm{min}(0.02 \mathrm{CV} / \mathrm{min})$ was then selected. For the HiTrap Protein A HP (5 ml, GE HealthCare, Uppsala, Sweden) different volumes $(1 \mathrm{ml}, 2 \mathrm{ml}$, $3 \mathrm{ml}$ and $5 \mathrm{ml}$ ) of either IVIg $(35.7 \mathrm{mg} / \mathrm{ml}$ ) or plasma were used. The buffer system used was PBS (running and washing buffer) for all columns. The volume of wash buffer (PBS) used was $12 \mathrm{ml}$ for all columns. Similarly, elution was performed identically for all columns using $12 \mathrm{ml}$ of $50 \mathrm{mM}$ citrate buffer ( $\mathrm{pH}$ 3.0). All experimental conditions were tested at least 3 times.

Human factor IX transgene expression. Human F.IX expression in mice sera was determined by ELISA according to a previously published protocol ${ }^{37}$.

Antibody titer determination. The anti-AAV8 IgG ELISA was previously described ${ }^{29}$. Briefly, plates were coated with AAV8 capsid $\left(1 \times 10^{10}\right.$ capsid particles $/ \mathrm{ml}, 50 \mu \mathrm{l}$ per well, unless indicated otherwise), blocked with PBS with 6\% non-fat dry milk, and serum or plasma samples added to the wells in duplicate. The levels of total IgG in plasma were determined by ELISA as previously described ${ }^{22}$. The in vitro AAV vector neutralizing antibody assay was performed as previously described ${ }^{52}$. The citrate buffer used to elute anti-AAV IgG bound to the column did not appear to influence the NAb assay performance.

Vector genome copy number determination. Tissues biopsies were taken from various organs. One biopsy per tissue was weighed and homogenized in 500-700 $\mu \mathrm{l}$ lysis buffer and genomic DNA was isolated according to the manufacturer's protocol (Puregene Blood Core kit, Qiagen, Hilden, Germany) and quantified using a Nanodrop ND-8000 spectrophotometer (Thermo Fisher Scientific, Courtaboeuf, France), $100 \mathrm{ng}$ of genomic DNA were used in each reaction in triplicate. Vector genome copy numbers per cell were quantified by real-time quantitative PCR as previously described ${ }^{39}$. Primers and probes used were as follows: huSEAP forward primer, 5'-CCTAGGTGGAGGCCGAAAG-3'; huSEAP reverse primer: 5'-TGGTCCCACCTTGGCTGTA-3'; huSEAP probe: $5^{\prime}$-AACCCCAGACCCTGAGTACCCAGATGA- ${ }^{\prime}$. The primers and probe to detect the AAV8-hF.IX vector genomes were previously described ${ }^{29}$.

Statistical analysis. Statistical analyses were performed using the Prism software version 7.0a (GraphPad Software, Inc., San Diego, CA). Statistical methods used to compare datasets are specified in the text. $p$ values below 0.05 were considered significant.

Data generated or analysed during this study are included in this published article (and its Supplementary Information files) or are available from the corresponding author on reasonable request.

Received: 7 October 2019; Accepted: 3 January 2020;

Published online: 21 January 2020

\section{References}

1. Mingozzi, F. \& High, K. A. Therapeutic in vivo gene transfer for genetic disease using AAV: progress and challenges. Nat. Rev. Genet. 12, 341-355 (2011). 10.1038/nrg2988.

2. Wang, D., Tai, P. W. L. \& Gao, G. Adeno-associated virus vector as a platform for gene therapy delivery. Nat. Rev. Drug. Discov. 18, 358-378, https://doi.org/10.1038/s41573-019-0012-9 (2019).

3. George, L. A. et al. Hemophilia B gene therapy with a high-specific-activity factor IX Variant. N. Engl. J. Med. 377, 2215-2227, https://doi.org/10.1056/NEJMoa1708538 (2017).

4. Maguire, A. M. et al. Safety and efficacy of gene transfer for Leber's congenital amaurosis. N. Engl. J. Med. 358, 2240-2248 (2008). NEJMoa0802315 [pii] 10.1056/NEJMoa0802315.

5. Manno, C. S. et al. Successful transduction of liver in hemophilia by AAV-Factor IX and limitations imposed by the host immune response. Nat Med 12, 342-347, https://doi.org/nm1358 [pii] 10.1038/nm1358 (2006).

6. Mendell, J. R. et al. Single-Dose Gene-Replacement Therapy for Spinal Muscular Atrophy. N. Engl. J. Med. 377, 1713-1722, https:// doi.org/10.1056/NEJMoa1706198 (2017).

7. Miesbach, W. et al. Gene therapy with adeno-associated virus vector 5-human factor IX in adults with hemophilia B. Blood. https:// doi.org/10.1182/blood-2017-09-804419 (2017).

8. Nathwani, A. C. et al. Long-term safety and efficacy of factor IX gene therapy in hemophilia B. N. Engl. J. Med. 371, 1994-2004, https://doi.org/10.1056/NEJMoa1407309 (2014).

9. Rangarajan, S. et al. AAV5-Factor VIII Gene Transfer in Severe Hemophilia A. N. Engl. J. Med. 377, 2519-2530, https://doi. org/10.1056/NEJMoa1708483 (2017).

10. Hoy, S. M. Onasemnogene Abeparvovec: First Global Approval. Drugs. https://doi.org/10.1007/s40265-019-01162-5 (2019).

11. Yla-Herttuala, S. Endgame: glybera finally recommended for approval as the first gene therapy drug in the European union. Mol. Ther. 20, 1831-1832, https://doi.org/10.1038/mt.2012.194 (2012).

12. Patel, U., Boucher, M., de Leseleuc, L. \& Visintini, S. In CADTH Issues in Emerging Health Technologies 1-11 (2016).

13. Colella, P., Ronzitti, G. \& Mingozzi, F. Emerging Issues in AAV-Mediated In Vivo Gene Therapy. Mol. Ther. Methods Clin. Dev. 8, 87-104, https://doi.org/10.1016/j.omtm.2017.11.007 (2018).

14. Calcedo, R. et al. Adeno-associated virus antibody profiles in newborns, children, and adolescents. Clin Vaccine Immunol 18, 15861588, https://doi.org/CVI.05107-11 [pii] 10.1128/CVI.05107-11 (2011). 
15. Erles, K., Sebokova, P. \& Schlehofer, J. R. Update on the prevalence of serum antibodies (IgG and IgM) to adeno-associated virus (AAV). J Med Virol 59, 406-411, https://doi.org/10.1002/(SICI)1096-9071(199911)59:3<406::AID-JMV22>3.0.CO;2-N [pii] (1999).

16. Li, C. et al. Neutralizing antibodies against adeno-associated virus examined prospectively in pediatric patients with hemophilia. Gene Ther, https://doi.org/:gt201190 [pii] 10.1038/gt.2011.90 (2011).

17. Masat, E., Pavani, G. \& Mingozzi, F. Humoral immunity to AAV vectors in gene therapy: challenges and potential solutions. Discov. Med. 15, 379-389 (2013).

18. Vandamme, C., Adjali, O. \& Mingozzi, F. Unraveling the Complex Story of Immune Responses to AAV Vectors Trial After Trial. Hum. Gene Ther. 28, 1061-1074, https://doi.org/10.1089/hum.2017.150 (2017).

19. Scallan, C. D. et al. Human immunoglobulin inhibits liver transduction by AAV vectors at low AAV2 neutralizing titers in SCID mice. Blood 107, 1810-1817, https://doi.org/10.1182/blood-2005-08-3229 (2006).

20. Jiang, H. et al. Effects of transient immunosuppression on adenoassociated, virus-mediated, liver-directed gene transfer in rhesus macaques and implications for human gene therapy. Blood 108, 3321-3328, https://doi.org/10.1182/blood-2006-04-017913 (2006).

21. Bortolussi, G. et al. Life-long correction of hyperbilirubinemia with a neonatal liver-specific AAV-mediated gene transfer in a lethal mouse model of Crigler-Najjar Syndrome. Hum. Gene Ther. 25, 844-855, https://doi.org/10.1089/hum.2013.233 (2014)

22. Boutin, S. et al. Prevalence of serum IgG and neutralizing factors against adeno-associated virus (AAV) types 1, 2, 5, 6, 8, and 9 in the healthy population: implications for gene therapy using AAV vectors. Hum. Gene Ther. 21, 704-712, https://doi.org/10.1089/ hum.2009.182 (2010).

23. de Back, D. Z., Neyrinck, M. M. \& Vrielink, H. Therapeutic plasma apheresis: Expertise and indications. Transfus. Apher. Sci. 58, 254-257, https://doi.org/10.1016/j.transci.2019.04.008 (2019).

24. Monteilhet, V. et al. A 10 Patient Case Report on the Impact of Plasmapheresis Upon Neutralizing Factors Against Adeno-associated Virus (AAV) Types 1, 2, 6, and 8. Mol. Ther. https://doi.org/10.1038/mt.2011.108 (2011).

25. Chicoine, L. et al. Plasmapheresis Eliminates the Negative Impact of AAV Antibodies on Microdystrophin Gene Expression Following Vascular Delivery. Mol. Ther. https://doi.org/10.1038/mt.2013.244 (2013).

26. Hurlbut, G. D. et al. Preexisting immunity and low expression in primates highlight translational challenges for liver-directed AAV8-mediated gene therapy. Mol. Ther. 18, 1983-1994, https://doi.org/10.1038/mt.2010.175 (2010).

27. Salas, D. et al. Immunoadsorption enables successful rAAV5-mediated repeated hepatic gene delivery in nonhuman primates. Blood Adv. 3, 2632-2641, https://doi.org/10.1182/bloodadvances.2019000380 (2019).

28. Kaplan, A. Complications of apheresis. Semin. Dial. 25, 152-158, https://doi.org/10.1111/j.1525-139x.2011.01026.x (2012).

29. Fitzpatrick, Z. et al. Influence of Pre-existing Anti-capsid Neutralizing and Binding Antibodies on AAV Vector Transduction. Mol. Ther. Methods Clin. Dev. 9, 119-129, https://doi.org/10.1016/j.omtm.2018.02.003 (2018).

30. Wang, L. et al. The pleiotropic effects of natural AAV infections on liver-directed gene transfer in macaques. Mol Ther 18, 126-134, doi:mt2009245 [pii] 10.1038/mt.2009.245 (2010)

31. Tzartos, S. J. et al. Antigen-specific apheresis of pathogenic autoantibodies from myasthenia gravis sera. Ann. N. Y. Acad. Sci. 1132, 291-299, https://doi.org/10.1196/annals.1405.017 (2008)

32. Rydberg, L., Bengtsson, A., Samuelsson, O., Nilsson, K. \& Breimer, M. E. In vitro assessment of a new ABO immunosorbent with synthetic carbohydrates attached to sepharose. Transpl. Int. 17, 666-672, https://doi.org/10.1007/s00147-004-0775-7 (2005).

33. Amital, H. et al. Antibodies against the VRT101 laminin epitope correlate with human SLE disease activity and can be removed by extracorporeal immunoadsorption. Rheumatol. 46, 1433-1437, https://doi.org/10.1093/rheumatology/kem181 (2007).

34. Pei, X. et al. AAV8 virions hijack serum proteins to increase hepatocyte binding for transduction enhancement. Virology $\mathbf{5 1 8}$, 95-102, https://doi.org/10.1016/j.virol.2018.02.007 (2018)

35. Wang, M. et al. Direct interaction of human serum proteins with AAV virions to enhance AAV transduction: immediate impact on clinical applications. Gene Ther. 24, 49-59, https://doi.org/10.1038/gt.2016.75 (2017).

36. Veron, P. et al. Humoral and cellular capsid-specific immune responses to adeno-associated virus type 1 in randomized healthy donors. J. Immunol. 188, 6418-6424, https://doi.org/10.4049/jimmunol.1200620 (2012).

37. Mingozzi, F. et al. Overcoming preexisting humoral immunity to AAV using capsid decoys. Sci. Transl. Med. 5, 194ra192, https://doi. org/10.1126/scitranslmed.3005795 (2013).

38. Corti, M. et al. Evaluation of Readministration of a Recombinant Adeno-Associated Virus Vector Expressing Acid AlphaGlucosidase in Pompe Disease: Preclinical to Clinical Planning. Hum. Gene Ther. Clin. Dev. 26, 185-193, https://doi.org/10.1089/ humc.2015.068 (2015).

39. Meliani, A. et al. Antigen-selective modulation of AAV immunogenicity with tolerogenic rapamycin nanoparticles enables successful vector re-administration. Nat. Commun. 9, 4098, https://doi.org/10.1038/s41467-018-06621-3 (2018).

40. Mingozzi, F. et al. Prevalence and pharmacological modulation of humoral immunity to AAV vectors in gene transfer to synovial tissue. Gene Ther. 20, 417-424, https://doi.org/10.1038/gt.2012.55 (2013).

41. Davidoff, A. M. et al. Purification of recombinant adeno-associated virus type 8 vectors by ion exchange chromatography generates clinical grade vector stock. J. Virol. Methods 121, 209-215, https://doi.org/10.1016/j.jviromet.2004.07.001 (2004).

42. Qu, G. et al. Separation of adeno-associated virus type 2 empty particles from genome containing vectors by anion-exchange column chromatography. J. Virol. Methods 140, 183-192, https://doi.org/10.1016/j.jviromet.2006.11.019 (2007).

43. Carrasco, C., Castellanos, M., de Pablo, P. J. \& Mateu, M. G. Manipulation of the mechanical properties of a virus by protein engineering. Proc. Natl Acad. Sci. USA 105, 4150-4155, https://doi.org/10.1073/pnas.0708017105 (2008).

44. Gao, G. P. et al. Novel adeno-associated viruses from rhesus monkeys as vectors for human gene therapy. Proc. Natl Acad. Sci. USA 99, 11854-11859, https://doi.org/10.1073/pnas.182412299 (2002).

45. Bambauer, R., Latza, R., Burgard, D. \& Schiel, R. Therapeutic Apheresis in Immunologic Renal and Neurological Diseases. Ther. Apher. Dial. 21, 6-21, https://doi.org/10.1111/1744-9987.12499 (2017).

46. Mingozzi, F. et al. Modulation of tolerance to the transgene product in a nonhuman primate model of AAV-mediated gene transfer to liver. Blood 110, 2334-2341, blood-2007-03-080093 [pii] 10.1182/blood-2007-03-080093 (2007).

47. Hui, D. J. et al. AAV capsid CD8 + T-cell epitopes are highly conserved across AAV serotypes. Mol. Ther. Methods Clin. Dev. 2, 15029, https://doi.org/10.1038/mtm.2015.29 (2015).

48. Mingozzi, F. et al. CD8(+) T-cell responses to adeno-associated virus capsid in humans. Nat. Med. 13, 419-422, https://doi. org/10.1038/nm1549 (2007)

49. Goyenvalle, A., Babbs, A., van Ommen, G. J., Garcia, L. \& Davies, K. E. Enhanced exon-skipping induced by U7 snRNA carrying a splicing silencer sequence: Promising tool for DMD therapy. Mol. Ther. 17, 1234-1240, https://doi.org/10.1038/mt.2009.113 (2009).

50. Virag, T., Cecchini, S. \& Kotin, R. M. Producing recombinant adeno-associated virus in foster cells: overcoming production limitations using a baculovirus-insect cell expression strategy. Hum. Gene Ther. 20, 807-817, https://doi.org/10.1089/hum.2009.092 (2009).

51. Ayuso, E. et al. High AAV vector purity results in serotype- and tissue-independent enhancement of transduction efficiency. Gene Ther 17, 503-510, doi:gt2009157 [pii] 10.1038/gt.2009.157 (2010).

52. Meliani, A. et al. Determination of anti-adeno-associated virus vector neutralizing antibody titer with an in vitro reporter system. Hum. Gene Ther. Methods. https://doi.org/10.1089/hgtb.2015.037 (2015). 


\section{Acknowledgements}

This work was supported by Genethon. It was also supported by the European Union, ERC-2013-CoG Consolidator Grant, grant agreement number 617432 (MoMAAV, to F.M.), European Union's research and innovation program under Grant Agreements No. 667751 (Myocure, to F.M.) and No. 755225 (CureCN, to F.M. and O.M.), E-Rare2 grant SMART-HaemoCare (to F.M.). This work was also supported by the "Investissements d'Avenir" programs managed by the ANR under reference ANR-11-INBS-0008 funding the Infectious Disease Models and Innovative Therapies (IDMIT, Fontenay-aux-Roses, France), and ANR-10-EQPX-02-01 funding the FlowCyTech facility (IDMIT, Fontenay-aux-Roses, France). We warmly thank all the members of ASW, FlowCyTech and L21 core lab facility from IDMIT center. All the data needed to evaluate the conclusions made in this paper are present within the data presented in the paper and/or the Supplemental Materials. Additional data may be requested from the authors.

\section{Author contributions}

B.B., P.V., C.L., J.-Y.D., S.M., Y.F., F.C., S.B., V.L., L.v.W., B.D. performed the experiments. P.V., O.M., F.M. wrote the manuscript. R.l.G., N.D.-B., O.B., P.M., C.M., O.M., F.M. conceived the study and directed the work.

\section{Competing interests}

O.M. and F.M. are inventors in patent describing methods to modulate immune responses to AAV and to prevent or remove anti-AAV antibodies. F.M. is employee and equity holder of Spark Therapeutics. P.M. is employee and equity holder of Ask Bio. C.M. is employee of Genosafe. O.M. is employee of Miltenyi Biotech. All other authors declare no competing interests.

\section{Additional information}

Supplementary information is available for this paper at https://doi.org/10.1038/s41598-020-57893-z.

Correspondence and requests for materials should be addressed to F.M.

Reprints and permissions information is available at www.nature.com/reprints.

Publisher's note Springer Nature remains neutral with regard to jurisdictional claims in published maps and institutional affiliations.

(c) (i) Open Access This article is licensed under a Creative Commons Attribution 4.0 International License, which permits use, sharing, adaptation, distribution and reproduction in any medium or format, as long as you give appropriate credit to the original author(s) and the source, provide a link to the Creative Commons license, and indicate if changes were made. The images or other third party material in this article are included in the article's Creative Commons license, unless indicated otherwise in a credit line to the material. If material is not included in the article's Creative Commons license and your intended use is not permitted by statutory regulation or exceeds the permitted use, you will need to obtain permission directly from the copyright holder. To view a copy of this license, visit http://creativecommons.org/licenses/by/4.0/.

(C) The Author(s) 2020 